Rufino Cabrera 1

Carlos Mayo ${ }^{1}$

Nicolás Suárez 1

César Infante 1

César Náquira 2

Marco Tulio A. García-Zapata ${ }^{3}$

\section{Conocimientos, actitudes y prácticas sobre la enfermedad de Chagas en población escolar de una zona endémica del Perú}

\author{
Knowledge, attitudes, and practices concerning \\ Chagas disease in schoolchildren from an endemic \\ area in Peru
}

1 Departamento de Ciencias Biológicas, Facultad de Ciencias, Universidad Nacional San Luis Gonzaga de Ica. Av. Los Maestros $s / n$, Casilla Postal 03, Ica, Perú. rcabrerach@hotmail.com

2 Oficina General de Asesoría Técnica, Centro Nacional de Laboratorios en Salud Pública, Instituto Nacional de Salud. Cápac Yupanqui 1400, Lima 11, Lima, Perú. cnaquira@ins.sld.pe

3 Instituto de Patologia Tropical e Saúde Pública, Universidade Federal de Goiás. C. P. 12911 Goiania, GO 74643-970, Brasil. zapata@iptsp.ufg.br mctulian1@hotmail.com

\begin{abstract}
This study analyzes knowledge, attitudes, and practices concerning Chagas disease among 241 primary schoolchildren in "La Tinguiña", Ica, Peru (December 2000 - January 2001). Less than 1\% of those interviewed knew that triatomines transmit Chagas disease, while nearly a quarter recognized the illness based on the appearance of "lumps" on the skin; 35.27\% knew that vector infestation is controlled using insecticides; $26.56 \%$ recognized the adult stage of the vector, and 21.16\% the nymphal instar; 14.11\% knew triatomines or "kissing bugs" by the name "chirimacha"; 82.57\% would accept an entomological survey, $66.80 \%$ would submit to a serological study, and $63.90 \%$ would participate in a triatomine search. The study shows that the population, despite having very limited knowledge on the disease and its vectors, shows interest in collaborating. Therefore, it is recommended that Chagas disease surveillance and control include educational programs and community participation.
\end{abstract}

Key words Chagas Disease; Knowledge, Attitudes, Practice; Surveillance; Students

Resumen El objetivo del estudio es presentar los resultados obtenidos sobre conocimientos, actitudes y prácticas acerca de la enfermedad de Chagas, en 241 escolares de educación primaria en La Tinguiña, Ica, Perú (diciembre 2000 - enero 2001). Menos del 1\% de los encuestados reconoce que los triatomas trasmiten la enfermedad de Chagas, y casi la cuarta parte reconoce la enfermedad por la formación de "ronchas" en la piel; el 35,27\% sabe que la infestación por el vector se controla con insecticidas. El 26,56\% reconoce a los estados adultos del vector y el 21,16\% a las ninfas; el 14,11\% lo conoce con el nombre de "chirimacha"; el 82,57\% aceptaría una encuesta entomológica; el 66,80\% permitiría un estudio serológico y el 63,90\% participaría en la búsqueda de triatominos. Este estudio revela que la población, a pesar de tener conocimientos muy limitados sobre la enfermedad y su vector, muestra interés en colaborar. Por lo tanto, se recomienda que las estrategias de vigilancia y control de esta enfermedad, incluyan necesariamente programas educativos y de participación comunitaria, en la implantación de futuros programas de control.

Palabras clave Enfermedad de Chagas; Conocimientos, Actitudes y Práctica; Vigilancia; Estudiantes 


\section{Introducción}

En América Latina la enfermedad de Chagas es una de las infecciones parasitarias de mayor importancia (Schumnis, 1994; WHO, 1991). En el Perú, las áreas endémicas están en la región sudoccidental que es la más importante (Herrer, 1977), y las regiones del norte y nororiental (MINSA, 2001). Hasta 1995, se habían notificado 1.679 casos (OPAS/OMS, 1996) y se estima que existen actualmente 24,170 casos de los cuales, 1.209 son agudos y 22,962 crónicos, la tasa de incidencia promedio anual es de 0,01$0,02 \times 100.000$ habitantes y la acumulada es de 7,29 x 100.000 casos (MINSA, 1998).

En la región sudoccidental se ha diseñado un plan de interrupción de la transmisión vectorial por Triatoma infestans, el único vector doméstico de esta parte del país; sin embargo, hasta la fecha sólo se han realizado rociamientos aislados o esporádicos como consecuencia de presiones sociales o políticas y poco o nada se ha hecho en materia de mejoramiento de vivienda o intervención educativa. Tampoco, se habían realizado estudios sociológicos sobre los conocimientos, actitudes y prácticas en poblaciones en riesgo, particularmente en los escolares, que permitan recoger información para replantear las estrategias de prevención y control. En otros países las poblaciones afectadas tienen conocimientos limitados (RojasArias, 2001; Schofield, 1985).

El objetivo del presente trabajo fue determinar el nivel de conocimientos, actitudes y prácticas sobre la enfermedad de Chagas y del vector en una población escolar de una zona endémica del departamento de Ica, Perú, como el reconocimiento del vector, sus hábitos, ecótopos, mecanismos de transmisión, síntomas y signos de la enfermedad, prácticas para prevenirla y evitar la colonización del vector, respuestas a las atenciones e intervenciones, fuentes de información, programas de dibujos animados, y la participación de los escolares en la vigilancia entomológica comunal.

\section{Material y métodos}

\section{Área de estudio}

El distrito de La Tinguiña, está ubicado al sur de la capital, Lima, a (140 01' 57" L.S) y a una altitud de 434 metros sobre el nivel del mar, su población reside en un área urbano-marginal y es una de las áreas endémicas del departamento de Ica (Cornejo et al., 1986; Villanueva, 1999). Las actividades económicas más importantes son la agricultura y el comercio. De acuerdo al Instituto Nacional de Estadística e Informática (INEI, 1994), el 1,8\% de su población reside en viviendas con características físicas inadecuadas, el $14,4 \%$ en condiciones de de hacinamiento y el $11,2 \%$ tienen alta dependencia económica.

\section{Población de estudio}

Estuvo constituida por 937 escolares del tercero al sexto grado de educación primaria que asistían regularmente a una escuela estatal de La Tinguiña, Ica. El centro educativo fue seleccionado por tener la población escolar más numerosa.

\section{Muestra}

Se calculó mediante un muestreo aleatorio una muestra representativa y significativa de 241 $(25,7 \%)$ del total de escolares asistentes del tercero al sexto grado de primaria entre diciembre de 2000 y enero de 2001, mediante el software Epi Info 6.04 versión en español, considerando un porcentaje hipotético de $40 \%$ de escolares que tenían conocimientos básicos, con un nivel de confianza al $99 \%$ y un margen de error de $\pm 0,03$.

\section{Criterios de selección}

Se incluyeron en el estudio a todos los escolares de ambos sexos que asistían regularmente a la escuela y que cursaban del tercero al sexto grado de educación primaria en el momento de la encuesta que además, residían en una localidad infestada o donde se habían detectado seropositivos a la enfermedad de Chagas.

\section{Cuestionario}

Como instrumento de investigación, se utilizó y aplicó un cuestionario ad hoc validado y precodificado que contenía 31 ítems elementales sobre la enfermedad de Chagas para los escolares de primaria como: mecanismos de transmisión, síntomas y signos, la biología del vector (hábitos, ecótopos), las actitudes frente a las intervenciones y las prácticas para prevenir la colonización del vector, fuentes de información, programas de dibujos animados y la participación de los escolares en la vigilancia entomológica comunal. Las preguntas se distribuyeron en: datos generales de los escolares (3), conocimientos de la enfermedad y del vector (11), actitudes (7) y prácticas (10). Del total, 10 fueron cerradas, una puntual y el resto de tipo abierta; fueron estructuradas en una secuen- 
cia no lógica para evitar la inducción de las respuestas por los escolares.

\section{Recolección de datos, clasificación} y evaluación de los cuestionarios

La información fue recogida por un supervisor y por 10 estudiantes (encuestadores) del último año de Biología de la Facultad de Ciencias de la Universidad Nacional San Luis Gonzaga de Ica, Perú, entrenados y seleccionados durante la validación de la encuesta. Las entrevistas se realizaron con la ayuda de muestrarios con estados adultos y ninfales de T. infestans, que fueron mostrados a los escolares durante la encuesta para comprobar la certeza de la respuesta; evitando mencionar la palabra "chirimacha" ni la frase "enfermedad de Chagas", para no influir en las respuestas; se anotaron aquellas que contestaron espontáneamente. Para cada encuesta se empleó entre 8-10 minutos.

Para la evaluación de la encuesta se elaboró una clave de respuestas y se asignó una puntuación proporcional a cada pregunta de acuerdo con su importancia y fueron calificadas por un investigador que conocía el tema. Para determinar el nivel de conocimientos se clasificaron en tres niveles: Insuficiente (I) con un puntaje de 0 a 50 puntos, Básico (II) de 51 a 75 puntos y Avanzado (III) de 76 a 100 puntos.

\section{Análisis estadístico}

Para el análisis se elaboró una base de datos, los cuales se procesaron mediante la estadística descriptiva con la ayuda del software Epi Info versión en español 6.04.

\section{Resultados}

\section{Edad y sexo de los escolares encuestados}

La edad de la población escolar estuvo entre 7-19 años $(X=10,39)$ con una desviación estándar de $\pm 1,72$. El 49,79\% (120) fueron varones y el $50,21 \%$ (121) mujeres.

\section{Nivel de conocimiento}

En la Tabla 1 se muestran los resultados acerca del Nivel de Conocimiento de los escolares sobre la enfermedad de Chagas y el vector, el $56,43 \%$ pertenecen al Nivel I (Insuficiente), el 39,83\% al Nivel II (Básico) y sólo el 3,74\% está comprendido en el Nivel III (Avanzado).

\begin{tabular}{|c|c|c|}
\hline \multicolumn{3}{|c|}{$\begin{array}{l}\text { Nivel de conocimiento sobre la enfermedad de Chagas y su vector } \\
\text { en población escolar del distrito de La Tinguiña, departamento de Ica, Perú, } \\
\text { Diciembre } 2000 \text {-Enero } 2001 .\end{array}$} \\
\hline Nivel & Número de escolares & $\%$ de escolares \\
\hline I - Insuficiente & 136 & 56,43 \\
\hline II - Básico & 96 & 39,83 \\
\hline III - Avanzado & 9 & 3,74 \\
\hline Total & 241 & 100,00 \\
\hline
\end{tabular}

\section{Conocimientos elementales}

En la Tabla 2 se presentan los resultados de las respuestas de los escolares sobre los conocimientos elementales de la enfermedad de Chagas y el vector; el $0,83 \%$ de los escolares conocen que los triatomas transmiten la enfermedad; respecto a los signos, el $24,07 \%$ lo reconoce por la formación de "ronchas" en la piel. El $14,11 \%$ respondió que, T. infestans, se llama "chirimacha", el 26,56\% identifica a los adultos y el $21,16 \%$ a las ninfas. Los ambientes más infestados son corrales $(18,67 \%)$ y dormitorios $(18,26 \%)$, las viviendas de adobe son las más colonizadas $(46,06 \%)$. Los sitios predilectos donde se refugian los triatominos son las grietas de las paredes $(29,88 \%)$; el $15,35 \%$ reconocen su hábito hematófago y consideraron que los perros son los más picados. El 35,27\% de los escolares saben que el vector se controla con la aplicación de insecticidas.

En la Tabla 3 se muestran las actitudes de los escolares frente a la enfermedad de Chagas y al vector, el $41,49 \%$ acudiría a un puesto de salud si son picados por el vector, el $37,34 \%$ acudiría a un hospital local; al explorar la posibilidad de realizar una encuesta entomológica en sus viviendas por parte del Ministerio de Salud, universidades u otras entidades, el 82,57\% la aceptaría. Al preguntarles si accedería a un examen serológico para descartar una infección el $66,80 \%$ de los entrevistados mostró su disposición al examen. El 63,90\% de los escolares manifestaron su deseo de participar en la búsqueda del vector en sus casas; asimismo, el $11,62 \%$ lo llevaría a un Veterinario si lo hallaran en sus viviendas. Ante la posibilidad de una intervención educativa sobre un problema de salud, el 90,87\% la aceptaría y el 51,87\% desearían recibirla en la escuela.

En la Tabla 4 se exponen los resultados relativos a las prácticas de los escolares respecto a la enfermedad y el vector, el 63,49\% admitieron 
Tabla 2

Conocimientos elementales sobre la enfermedad de Chagas y del vector en población escolar del distrito de La Tinguiña, departamento de Ica, Perú, Diciembre 2000-Enero 2001.

\begin{tabular}{|c|c|c|}
\hline Respuesta de los escolares & $\mathbf{n}$ & $\%$ \\
\hline El mal de Chagas es trasmitido por triatomas & 2 & 0,83 \\
\hline La enfermedad se reconoce por ronchas en la piel & 58 & 24,07 \\
\hline La enfermedad se reconoce por formación de heridas en la piel & 14 & 5,81 \\
\hline El nombre común del vector es "chirimacha" & 34 & 14,11 \\
\hline Reconoce a los adultos de T. infestans & 64 & 26,56 \\
\hline Reconoce a los estados ninfales & 51 & 21,16 \\
\hline \multicolumn{3}{|l|}{ Los ambientes que el vector infesta son: } \\
\hline Corrales & 45 & 18,67 \\
\hline Dormitorios & 44 & 18,26 \\
\hline Cocinas & 21 & 8,71 \\
\hline El vector coloniza viviendas de adobe & 111 & 46,06 \\
\hline El vector coloniza viviendas de material noble & 24 & 9,96 \\
\hline El vector coloniza viviendas de estera/quincha & 10 & 4,15 \\
\hline \multicolumn{3}{|l|}{ Los sitios donde se refugia el vector son: } \\
\hline Grietas de paredes & 72 & 29,88 \\
\hline Detrás de muebles, almanaques, entre las ropas, etc & 30 & 12,45 \\
\hline Entre las cañas o maderas en los techos & 12 & 4,98 \\
\hline El vector se alimenta de sangre de animales y del hombre & 37 & 15,35 \\
\hline El animal doméstico más picado por el vector es el perro & 37 & 15,35 \\
\hline \multicolumn{3}{|l|}{ Las medidas que deben aplicarse para controlar el vector son: } \\
\hline Aplicar insecticidas o "veneno" & 85 & 35,27 \\
\hline Incinerarlos con queroseno o matarlos por otros procedimientos & 41 & 17,01 \\
\hline Limpieza de los ambientes domésticos y peridomésticos & 16 & 6,64 \\
\hline Enlucir las paredes de las viviendas & 1 & 0,41 \\
\hline
\end{tabular}

tener almanaques $\mathrm{u}$ otros papeles en las paredes de sus dormitorios, el 88,79\% cría animales domésticos en sus viviendas y el animal más frecuentemente criado el perro $(39,00 \%)$.

$\mathrm{Al}$ explorar sobre la limpieza, el promedio de barridos de casa por día es 2,3. Respecto a los medios de comunicación, la televisión es la más utilizada $(63,07 \%)$, pero los más vistos son los canales nacionales, las emisoras radiales más escuchadas son locales. El 7,88\% de los entrevistados había escuchado hablar a sus profesores alguna vez sobre la enfermedad, aunque no todos recordaron su nombre, finalmente el $17,84 \%$ de los escolares habían visto al vector en sus casas en los últimos 3 meses al estudio.

\section{Discusión}

La encuesta en población escolar de una zona endémica de Ica, Perú, reveló que el nivel de conocimientos sobre aspectos elementales de la enfermedad de Chagas son limitados, simi- lares a los obtenidos en alumnos de áreas rurales epidemiológicamente diferentes en Argentina (Sanmartino \& Crocco, 2000).

Más de la mitad de los encuestados tienen un nivel Insuficiente (I), es decir no conocen los aspectos elementales sobre la enfermedad de Chagas y el vector; el 39,83\% tienen un nivel Básico (II) y escasamente el 3,74\% tienen conocimientos Avanzados (III), lo que revela que es imprescindible realizar una intervención educativa en esta población sobre el problema.

Menos del 1\% de los entrevistados reconocieron el rol vectorial de T. infestans en la transmisión de la enfermedad, y asimismo no sabían las otras vías, que los expone al riesgo de adquirir la infección por las mismas, respecto a este ítem nuestros resultados son menores a los hallados en alumnos de escuelas rurales en Argentina, donde también el conocimiento de los otros modos de transmisión es muy limitado (Sanmartino \& Crocco, 2000); sin embargo, es similar al nivel de conocimiento en poblaciones de adultos en áreas endémicas con actividades de control y sin control de Honduras (Ávila et al., 1998), al de una comunidad de Chuquisaca, Bolivia (Caballero-Zamora \& De Muynck, 1999) y también a una población endémica en Mambaí, Brasil (Bizerra et al., 1981). La mayoría de las poblaciones endémicas no asocian al vector con la enfermedad de Chagas. En la población adulta endémica de Posse, Estado de Goiás, Brasil, con más de una década bajo control, el 72,88\% de los entrevistados atribuyeron como causa de la enfermedad de Chagas a los triatominos (Williams-Blangero et al., 1999), que explicaría la eficacia de la intervención educativa.

La formación de los chagomas no son reconocidos por la mayoría de los escolares, demostrándose el limitado conocimiento sobre el aspecto clínico, a diferencia de los jefes de familia en Mambaí, Brasil, que conocen mejor y consideran que la formación de heridas en la piel es una característica de la enfermedad (Bizerra et al., 1981). Los indios bolivianos también describen mejor el aspecto clínico (Caballero-Zamora \& De Muynck, 1999), disimilitud que se explicaría debido a que estas poblaciones a diferencia de los escolares proceden de zonas con alta endemicidad y han convivido más tiempo con el vector y la enfermedad.

El 14,11\% de los entrevistados respondieron que el nombre común de $T$. infestans es "chirimacha”, el $26,56 \%$ reconocieron a los adultos, pero sólo el $21,16 \%$ a las ninfas; por ello, debería incluirse brevemente el ciclo vital completo del vector en el currículo escolar y en las intervenciones educativas activas (Bizerra et al. 
1981). Un buen porcentaje de los escolares están familiarizados con el vector, pero no conocen el rol en la transmisión de la enfermedad. Resultado relativamente superior al obtenido en menores de 20 años en una aldea rural del Municipio de Luz en Minas Gerais, Brasil (Dias \& Dias, 1982), pero inferior al hallazgo en Honduras (Ávila et al., 1998), esta diferencia se debería a que los escolares encuestados viven en un área urbano-marginal, donde los indicadores entomológicos no son muy altos y la enfermedad no es frecuente (Villanueva, 1999), por lo tanto, tienen un conocimiento más limitado, como se ha comentado anteriormente.

El ecótopo y los hábitos del vector como la infestación de los ambientes de la vivienda, la colonización de los tipos de vivienda y el reconocimiento de los refugios del vector son mejor conocidos por los entrevistados con excepción de su hábito hematófago. Las poblaciones endémicas de Honduras (Ávila et al., 1998) y de Bolivia (Caballero-Zamora \& De Muynck, 1999), tienen un nivel de conocimiento más elevado sobre aspectos ecológicos del vector. Las poblaciones en riesgo deben conocer estos aspectos del vector para su aplicación en el control y la vigilancia entomológica comunal.

En relación con las medidas de prevención y control, el 35,27\% de los entrevistados consideró que la infestación por el vector se controla con la aplicación de insecticidas, conocidos comúnmente por los escolares como "veneno", un menor porcentaje consideró que deberían incinerarlos o matarlos utilizando otros procedimientos; sin embargo, la limpieza de las viviendas y el enlucido de las paredes son medidas de control pobremente conocidas. El nivel de conocimiento respecto a estos ítems son inferiores a aquellos de población adulta de Honduras (Ávila et al., 1998), las comunidades bolivianas utilizan ahumados de plantas, insecticidas y otros productos como queroseno para controlar los vectores similares a nuestros hallazgos (Caballero-Zamora \& De Muynck, 1999). Aunque no se han obtenido datos de prácticas tradicionales en el control del vector; se sabe, sin embargo, que en la provincia de Nazca (Ica) la población utiliza el ahumado de Capsicum spp. "ají" solanáceas para controlar el vector, práctica que debe ser considerada como complemento del control químico. Además, se requiere que las otras medidas de control no vectorial deben ser enfatizadas, ya que la sola aplicación de insecticidas sin considerar el mejoramiento de la vivienda y la participación de la comunidad en mantener una vivienda higiénica no eliminará ni evitará la colonización por el vector.

\begin{tabular}{|c|c|c|}
\hline Respuesta de los escolares a las actitudes & $\mathbf{n}$ & $\%$ \\
\hline \multicolumn{3}{|l|}{ Si es picado por el vector acudiría a: } \\
\hline Un puesto de salud & 100 & 41,49 \\
\hline Un hospital local & 90 & 37,34 \\
\hline Un doctor & 12 & 4,98 \\
\hline Un familiar de mayor edad & 10 & 4,15 \\
\hline $\begin{array}{l}\text { Aceptaría una encuesta entomológica por el Ministerio } \\
\text { de Salud, universidades u otras entidades }\end{array}$ & 199 & 82,57 \\
\hline Aceptaría un examen serológico para descartar una infección & 161 & 66,80 \\
\hline Participaría en la búsqueda de triatominos en sus casas & 154 & 63,90 \\
\hline \multicolumn{3}{|l|}{ Si encuentra al vector en sus casas lo llevaría: } \\
\hline A un veterinario & 28 & 11,62 \\
\hline Al hospital & 21 & 8,71 \\
\hline Al médico & 14 & 5,81 \\
\hline A la universidad & 5 & 2,07 \\
\hline Aceptaría una intervención educativa sobre un problema de salud & 219 & 90,87 \\
\hline \multicolumn{3}{|l|}{ La intervención educativa los recibiría en: } \\
\hline La escuela & 125 & 51,87 \\
\hline En sus casas & 46 & 19,09 \\
\hline En un establecimiento de salud & 20 & 8,29 \\
\hline
\end{tabular}

Ante la posible picadura del vector un porcentaje considerable de escolares tienen una actitud correcta porque acudirían a los establecimientos de salud que permitiría su atención oportuna. Existe una buena disposición para aceptar una encuesta entomológica por el Ministerio de Salud o por otras entidades; sin embargo, se ha observado que la población adulta tiene un nivel de aceptación más bajo debido, probablemente, a las repetidas encuestas y a la escasa atención del problema, en la zona de estudio no se han realizado rociamientos en los últimos 10 años. Por otro lado, la aceptabilidad para someterse a un examen serológico no es muy alta; lo que probablemente refleja la creencia de la comercialización de la sangre, aspecto que debería tomarse en cuenta para sensibilizar a la población con el fin de hacerles conocer la importancia de la muestra sanguínea en el diagnóstico de la infección.

La aceptabilidad de los escolares respecto a su participación en la búsqueda de los triatominos en sus casas es relativamente alta, este ítem no había sido evaluado en estudios sociales previos a pesar de su importancia. La participación de los escolares en la vigilancia entomológica comunal de T. infestans, podría ser una excelente alternativa debido a que los niños tienen una gran habilidad para capturar 
Tabla 4

Prácticas de una población escolar del distrito de La Tinguiña, departamento de Ica, Perú, respecto a la enfermedad de Chagas y al vector, Diciembre 2000-Enero 2001.

\begin{tabular}{lrc}
\hline Respuesta sobre prácticas & $\mathbf{n}$ & $\%$ \\
\hline Tiene almanaques o papeles en las paredes de sus dormitorios & 153 & 63,49 \\
Cría animales domésticos en su vivienda & 214 & 88,79 \\
El animal doméstico que frecuentemente crían es el perro & 94 & 39,00 \\
El número promedio de barridos diarios de las casas es & 2,3 & $1,003^{*}$ \\
Los medios de comunicación más usados son: & 152 & 63,07 \\
La televisión & 73 & 30,29 \\
La radio & 13 & 5,39 \\
El periódico & 98 & 40,66 \\
Se informan a través del canal "América televisión" & 34 & 14,11 \\
Se informan a través del canal "Panamericana televisión" & & \\
Las emisoras radiales más escuchadas son: & 15 & 6,22 \\
Radio Mega Star & 11 & 4,56 \\
Radio Pacífico y Radio K-Buena & & \\
Los programas de dibujos animados más vistos son: & 90 & 37,34 \\
Digimon & 39 & 16,2 \\
Pokemon & 31 & 12,18 \\
Dragon Ball & 19 & 7,88 \\
Escuchó hablar a sus profesores sobre enfermedad de Chagas & 43 & 17,84 \\
Tres meses antes del estudio encontró al vector en su vivienda & 43 \\
\hline * DS Desviación estándar & &
\end{tabular}

* DS = Desviación estándar
La práctica de riesgo de mantener almanaques o papeles en las paredes de sus dormitorios, favorece la colonización del vector debido a que los insectos lo usan como refugio, como ocurre en la mayoría de las viviendas de adobe sin enlucir en las áreas rurales y menos frecuente en las zonas urbano-marginales.

La mayoría de los encuestados cría animales domésticos y el perro es el más frecuente, esta práctica también favorece la colonización de la vivienda y permite mantener altas densidades de triatominos, pues sirven como fuente de alimentación al vector o como reservorios del parásito. Resultados similares a los nuestros se han reportado en áreas rurales de Argentina (Sanmartino \& Crocco, 2000), los nativos bolivianos, creen que donde hay más animales la densidad triatomínica es alta (Caballero-Zamora \& De Muynck, 1999). Los animales domésticos juegan un rol importante en el ciclo de trasmisión doméstica de la enfermedad (Minter, 1978). En La Tinguiña se ha identificado al perro como el reservorio más importante de T. cruzi (Fernández \& Quicaño, 1995). En las estrategias de intervención educativa se debe recomendar la crianza de animales en ambientes a prueba de triatominos y fuera de la vivienda, y debe resaltarse el papel de los mamíferos como reservorios de la enfermedad, como aspectos elementales dentro del ordenamiento ambiental.

La deficiente limpieza de la vivienda, demuestra que debería sensibilizarse a la población sobre la importancia que tiene la limpieza de la vivienda y del peridomicilio en la colonización del vector (Dias, 1998). En las viviendas desordenadas y sucias el vector se encuentra con mayor frecuencia (Sanmartino \& Crocco, 2000).

Los canales televisión más vistos tienen señal a nivel nacional, mientras que las emisoras radiales más escuchadas son locales y muy pocos utilizan los medios escritos. En base a estos resultados se recomienda difundir aspectos elementales de la enfermedad y propiciar la participación comunitaria a través de la televisión nacional y las radios locales para tener éxito en la sensibilización (Schofield, 1985). Los niños son los mejores portavoces y divulgadores de un programa de control en una comunidad, al actuar como multiplicadores (García-Zapata, 1991). El uso de folletos educativos fue el medio de difusión más efectiva en el Brasil asociado a un programa educativo en las escuelas (García-Zapata, 1985), en nuestro país se deben validar los instrumentos educativos antes de su aplicación para incrementar la eficacia de la intervención. 
Frente al panorama actual del control de la transmisión vectorial de la enfermedad de Chagas en la Macrorregión sur del Perú, la participación comunitaria de los escolares en la vigilancia entomológica previa capacitación es una gran alternativa que permitiría una vigilancia sostenible en el tiempo y de bajo de costo. La vigilancia comunal no está bien estructurada en el país, por lo que se espera que las localidades rociadas se reinfesten con facilidad; por ello, la vigilancia entomológica es la etapa más importante y la más difícil de sostener en el tiempo (Dias, 1987; García-Zapata \& Marsden, 1994). Por esta razón, se requiere de la decisión política del gobierno (Días, 2001) para hacer realidad el control de este problema en el país. Finalmente, se demuestra la necesidad de desarrollar estudios cualitativos sobre estos y otros aspectos con el propósito de conocer mejor la concepción de la enfermedad de Chagas por la comunidad.

\section{Referencias}

ÁVILA, G. M.; MARTINEZ, H. M.; PONCE, C.; PONCE, E. \& SOTO, H. R., 1998. La enfermedad de Chagas en la zona central de Honduras: Conocimientos, creencias y prácticas. Revista Panamericana de Salud Pública, 3:158-163.

BIZERRA, J. F.; GAZZANA, M. R.; COSTA, C. H.; MELLO, D. A. \& MARSDEN, P. D., 1981. A survey of what people know about Chagas' disease. World Health Forum, 2:394-397.

BRICEÑO-LEON, R., 1996. Siete tesis sobre la educación sanitaria para la participación comunitaria. Cadernos de Saúde Pública, 12:7-30.

CABALLERO-ZAMORA, A. \& DE MUYNCK, A., 1999. Actitudes y creencias de los indios quechuas de la provincia Zudañez, departamento de Chuquisaca, Bolivia, frente al vector de la enfermedad de Chagas. In: Chagas, la Enfermedad en Bolivia. Conocimientos al Inicio del Programa de Control (1998-2002) (J. R. A. Cassab, F. Noireau \& G. Guillén, org.), pp. 171-197, La Paz: Ministerio de Salud y Previsión Social/Organización Panamericana de la Salud/Organización Mundial de la Salud/Institut de Recherche pour le Developpement/Instituto Boliviano de Biología de Altura.

CORNEJO, S. J.; CORNEJO, D. A.; CUBAS, N. E.; SOLIS, A. H.; ESPINOZA, B. Y.; ALBUQUERQUE, A. M.; NAUPAY, A. \& ATENCIA, V. A., 1986. Epidemiología de la enfermedad de Chagas en el departamento de Ica. I. Estudio del vector y reservorio humano. Boletín de Medicina Tropical, 5:10-13.

DIAS, J. C. P., 1987. Control of Chagas disease in Brazil. Parasitology Today, 3:336-341.

DIAS, J. C. P., 1998. Problemas e possibilidades de participação comunitária no controle das grandes endemias no Brasil. Cadernos de Saúde Pública, 14(Sup.2):19-37.
DIAS, J. C. P., 2001. Doença de Chagas, ambiente, participação e Estado. Cadernos de Saúde Pública, 17 (Sup.):165-169.

DIAS, J. C. P. \& DIAS, R. B., 1982. Las viviendas y la lucha contra los vectores de la enfermedad de Chagas en el hombre, en el Estado de Minas Gerais, Brasil. Boletín de la Oficina Sanitaria Panamericana, 93:453-467.

FERNANDEZ, R. M. O. \& QUICAÑO, A. N. Y., 1995. Reservorios Domésticos de Trypanosoma cruzi en el Distrito de La Tinguiña-Ica. Tesis de Licenciatura, Ica: Facultad de Ciencias, Universidad Nacional San Luis Gonzaga de Ica.

GARCIA-ZAPATA, M. T. A., 1985. Vigilância Epidemiológica no Controle do Triatoma infestans em Duas Áreas no Estado de Goiás-Brasil. Dissertação de Mestrado, Brasilia: Facultade de Ciências da Saúde, Universidade de Brasília.

GARCIA-ZAPATA, M. T. A., 1991. A divulgação científica no controle de doenças tropicais: Um punto de vista. Comunicação e Sociedade, 10:103-114.

GARCIA-ZAPATA, M. T. A. \& MARSDEN, P., 1994. Enfermedad de Chagas: Control y vigilancia con insecticidas y participación comunitaria en Mambaí, Goiás, Brasil. Boletín de la Oficina Sanitaria Panamericana, 116:97-110.

GARCIA-ZAPATA, M. T. A.; MARSDEN, P.; VIRGENS, D. \& SOARES, V. A., 1988. Epidemiological vigilance with community participation in the control of vectors of Chagas Disease in Goiás, Central Brazil. Revista de Microbiología, 20:106-117.

HERRER, A., 1977. Reseña de la entomología médica en el Perú. I. Principales aspectos entomológicos en la bartonelosis, la trypanosomiasis y la leishmaniasis. Revista Peruana de Entomología, 20:19-24. INEI (Instituto Nacional de Estadística e Informática)/ 
UNFPA (Fondo de Población de las Naciones Unidas), 1994. Perú: Mapa de Necesidades Básicas Insatisfechas de los Hogares a Nivel Distrital. Dirección Técnica de Demografía y Estudios Sociales. Lima: Editora Gráfica Tricolor.

MINSA (Ministerio de Salud), 1998. Doctrina, Normas y Procedimientos para el Control de la Tripanosomiosis o Enfermedad de Chagas en el Perú. Dirección General de Salud de las Personas. Programa de Control de Malaria y Otras Enfermedades Metaxénicas. Lima: Minsa.

MINSA (Ministerio de Salud), 2001. Enfermedad de Chagas. Módulos Técnicos. Serie de Documentos Monográficos 12. Lima: MINSA/Oficina General de Epidemiología, Instituto Nacional de Salud.

MINTER, D. M., 1978. Efectos de la presencia de animales domésticos en viviendas infestadas sobre la transmisión de la enfermedad de Chagas al hombre. Boletín de la Oficina Sanitaria Panamericana, 84:332-343.

OPAS (Organizacão Pan-Americana da Saúde)/OMS (Organização Mundial da Saúde), 1996. Iniciativa del Cono Sur, V Reunión de la Comisión Intergubernamental para la Eliminación del Triatoma infestans y la Interrupción de la Tripanosomiasis Americana Transfusional. Porto Alegre: OPAS/ OMS. (mimeo.)

PETANA, W., 1976. Educación para el control de la enfermedad de Chagas. Boletín de la Oficina Sanitaria Panamericana, 31:50-56.
ROJAS-DE-ARIAS, A., 2001. Chagas disease prevention through improved housing using an ecosystem approach to health. Cadernos de Saúde Pública, 17 (Sup.):89-97.

SANMARTINO, M. \& CROCCO, L., 2000. Conocimientos sobre la enfermedad de Chagas y factores de riesgo en comunidades epidemiológicamente diferentes de Argentina. Revista Panamericana de Salud Pública, 7: 173-178.

SCHMUNIS, A. G., 1994. La Tripanosomiasis americana como problema de salud pública. In: La Enfermedad de Chagas y el Sistema Nervioso (Organización Panamericana de la Salud, org.), pp. 331, Publicación Científica 547, Washington, DC: Organización Panamericana de la Salud.

SCHOFIELD, C. J., 1985. Control of Chagas' disease vectors. British Medical Bulletin, 41:187-194.

VILLANUEVA, C., 1999. Aspectos epidemiológicos de la trypanosomiosis americana en el departamento de Ica - Perú 1945-1995. Revista Peruana de Parasitología, 14:87-98.

WILLIAMS-BLANGERO, S.; VANDERBERG, J. L. \& TEIXEIRA, A. R. L., 1999. Attitudes towards Chagas' disease in an endemic Brazilian community. Cadernos de Saúde Pública, 15:7-13.

WHO (World Health Organization), 1991. Control of Chagas Disease. WHO Technical Report Series 811. Geneva: WHO.

Recibido el 7 de enero de 2002

Versión final presentada el 29 de abril de 2002

Aprobado el 15 de julio de 2002 